\title{
1 Significance of healthy viscous dietary fibres on the performance of gluten-free
}

2 rice-based formulated breads.

3

4 Sandra Pérez-Quirce ${ }^{1}$, Concha Collar ${ }^{2}$, Felicidad Ronda ${ }^{1 *}$

5

6 (1) Department of Agriculture and Forestry Engineering, Food Technology, College of

7 Agricultural and Forestry Engineering, University of Valladolid, Av. Madrid, 44, 34004

8 Palencia, Spain.

9 (2) Cereal Group, Food Science Department, Instituto de Agroquímica y Tecnología de

10 Alimentos, IATA-CSIC, Avda. Catedrático Agustín Escardino 7, 46980 Paterna, Spain.

12 *Corresponding author: Felicidad Ronda. Tel : 34979108339 ; E-mail:

13 fronda@iaf.uva.es 


\section{Abstract}

The impact of associated viscous dietary fibers (hydroxypropylmethylcellulose semifirm -SFE- and weak -NE- gel forming, and barley ß-glucan, BBG) incorporated at different amounts (1.6-7.5\%, flour basis) into gluten-free rice-based dough formulations, on the breadmaking performance and staling behaviour of hydrated (70$110 \%$, flour basis) fibre-flour composite blends has been investigated.

Single BBG addition fails to mimic gluten visco-elasticity properly, but simultaneous incorporation of either SFE or NE contribute to bread improvement in terms of bigger volume and smoother crumb. $-3.3-\mathrm{g}$ of $\mathrm{BBG}(70 \%$ purity) and $104 \mathrm{~mL}$ of water addition to $100 \mathrm{-g}$ rice flour provided sensorially accepted breads $(7.6 / \underline{109})$ with a theoretical $-\beta$-glucan content of $1.24-\mathrm{g} / 100-\mathrm{g}$ GF bread that would allow a daily $\beta$ glucan intake of $3 \mathrm{~g}$ withprovided a bread consumption of $240 \mathrm{~g} /$ day. A daily intake of $240 \mathrm{~g}$ of BBG-nriched GF bread (four servings) is high enough to meet the requirements of the EFSA health claim (3 g/day), contributing a reduced blood cholesterol levelComplementary tests should be carried out to testknow the amount and molecular weight of $\beta$-glucan in the final bread before assuring the nutritional benefit of this addition.

Keywords: Gluten-free; Hydrocolloids; $\beta$-glucan; bread quality 


\section{Introduction}

42

The increased consumer demand for healthy foods has driven to address concerted efforts from both research and industry to develop breads that combine properly health benefits with good physico-chemical and sensory properties. This goal is specially challenging in gluten-free (GF) breadmaking where the lack of gluten biopolymer seriously constrains dough visco-elastic character, leads to a failure in carbon dioxide entrapment, and hence deteriorates the techno-functional quality of resulting breads. In addition, a poor nutritional balance often characterises the multi-ingredient GF matrices (Thomson 2009). GF baked goods are often low in fibre, both soluble and insoluble; consequently its enrichment with dietary fibre seems to be necessary (Sabanis et al. 2009).

The natural, synthetic and biotechnological hydrocolloids, because of their high waterbinding capacity and their structure-creating behaviour, are mostly used in the different recipes for replacing the gluten network and its functionality (Houben, Hoechstoetter, and Becker 2012). Water availability plays a crucial role in the functionality of hydrocolloids by binding to the macromolecules in three different ways: via hydrogen bounds, embedded in inter- or intramolecular openings or immobilized by structuring (Anton 2008). The modified cellulose derivative hydroxypropyl-methyl-cellulose HPMC- (linear and neutral polymer) has, because of its hydrophilic character, a high water-binding capacity and also has, in its structure, hydrophobic methyl and hydrophilic hydroxypropyl groups located, which makes HPMC an interface activity in 

boundaries of the expanding gas cells. During baking, the gas-binding capacity is

66 67 increased and higher volume can be achieved ((Bell 1990; Collar et al 1999). The positive effects of isolates of cereal $\beta$-glucans have been recently reviewed by Wood (2010), with most of the data deriving from studies with oat $\beta$-glucans, followed by barley and rye (Kinner et al 2011). The European Food Safety Authority (EFSA) has recently approved health claims for foods that contribute to the diet $3 \mathrm{~g}$ per day of $\beta$ glucan (BG) from oat or barley for its ability to reduce the LDL-cholesterol level in blood, and for foods that provide at least $4 \mathrm{~g} / 30 \mathrm{~g}$ carbohydrate, for reducing the postprandial glycaemic response (EFSA 2011). Isolates of cereal ß-glucan are hydrocolloids with thickening properties (Lazaridou et al 2007) that could replace or supplement the action of HPMC when added in appropriate amounts, besides increasing nutritional value of GF bread in terms of dietary fiber content with proven health promoting effects. It is also stressed that high concentration of $\beta$-glucan decreases the water availability for the protein network and thus impairs the baking properties of wheat breads (Gill et al 2002). Molecular mass and structure, chain length, bonds and chemical modification, added doses, raw materials and process parameters used - $\mathrm{pH}$ value, temperature, shearing, ionic bonds and the attendance of ions- account for some factors determining the significance of associated hydrocolloids in bread performance (Houben, Hoechstoetter, and Becker 2012).

Previous studies haves shown a great difference in the effect of $ß$-glucan and HPMC of different gel strengths on gluten-free rice dough viscoelastic behaviour (Collar et al 1999; Collar, Santos, and Rosell 2005; Collar, Santos, and Rosell 2007; Ronda et al 2013). The water competition of the fibre macromolecules expliciting different water binding and gelling abilities resulted in additive, synergistic and/or antagonistic effects 
111 (Megazyme ${ }^{\circledR}$ kit); $140 \mathrm{kDa}$ molecular weight (size exclusion HPLC). BBG extract was

112 analysed for gluten content and a concentration under the detection limit was obtained

$113 \mid(<6.2 \mathrm{mg} / \mathrm{kg}$ of gGluten $)$ using the ELISA test based on the R5 antibody. 
115 A straight dough process was performed using the following formula on a $100 \mathrm{~g}$ rice 116 flour basis: $6 \%$ oil, $5 \%$ sucrose, $2 \%$ salt, $3 \%$ dried yeast, and $70 \%$ water. Combinations 117 of fibres according to a Draper-Lin small composite design for sampling (Draper and 118 Lin 1990) were added to the basic formula at different hydration levels (Table 1).

119 Design factors (quantitative independent factors) tested at five levels $(-1.4142,-1,0,1$, 120 1.4142), included SFE (from 0.10 to $2.50 \mathrm{~g} / 100 \mathrm{~g}$ flour basis), NE (from 0.10 to $2.50 \mathrm{~g}$ $121 / 100 \mathrm{~g}$ flour basis), BBG (from 0.10 to $3.90 \mathrm{~g} / 100 \mathrm{~g}$ flour basis), and WATER (from 0 122 to $40 \mathrm{~mL}$ extra water with respect to $70 \mathrm{~mL} / 100 \mathrm{~g}$ flour basis, that was the minimum $\underline{\text { amount added) }}{ }_{-}--$The model resulted in 19 different combinations of fibre-enriched hydrated rice-based doughs, including three central point replicates. These replicates were made in order to know the repeatability and accuracy of results. GF dough-making was achieved by blending first solid ingredients in a kitchen-aid professional mixer 127 (KPM5) for $10 \mathrm{~s}$ at speed 2. Then, liquid ingredients (oil and water at $20 \pm 2{ }^{\circ} \mathrm{C}$ ) were 128 added and mixed for 5 min at speed 6 . The dough, $200 \mathrm{~g}$, was placed into an alumnium oil coated pan and was proofed at $30^{\circ} \mathrm{C}$ and $(-90 \pm 5) \%$ relative moisture for $40 \mathrm{~min}$. Subsequently, baking was carried out in a Salva oven (Lezo, Spain) at $190^{\circ} \mathrm{C}$ for 40

131 min. After baking, breads were removed from the pan and left for one hour at room

132 temperature before analysis. To study the effect on staling, breads were stored for 0,1 , 2, 5, 7 and 9 days at $(4 \pm 2)^{\circ} \mathrm{C}$. To study the effect on staling, breads were stored in hermetic polypropylene bags for $0,1,2,5,7$ and 9 days at $(4 \pm 2){ }^{\circ} \mathrm{C}$. Seven breads were made in eachper run (one batch).

2.3. Evaluation of bread quality 
138 Bread volume was determined in duplicate using a volume analyzer BVM-L370 TexVol

139 Instruments (Viken, Sweden). The bread was weighed immediately after removal from

140 the pan once cooled. A digital calibre was used to measure bread height and width.

141 Crumb texture was determined in triplicate with a TA-XT2 texture analyser (Stable

142 Microsystems, Surrey, UK) provided with the software "Texture Expert". An

143 Aluminium $20 \mathrm{~mm}$ diameter cylindrical probe was used in a "Texture Profile Analysis"

144 double compression test (TPA) to penetrate to $50 \%$ depth, at $1 \mathrm{~mm} / \mathrm{s}$ speed test, with a

14530 s delay between first and second compression (Collar, Bollain, and Angioloni 2005).

146 Hardness $(\mathrm{N})$, chewiness $(\mathrm{N})$, cohesiveness, springiness and resilience were calculated

147 from the TPA graphic. Analysis was carried out at $(20 \pm 2){ }^{\circ} \mathrm{C}$ for bread slices of $20 \mathrm{~mm}$

148 thickness taken from the centre of the loaf.

149 Colour was measured with a Minolta spectrophotometer CN-508i (Minolta, Co.LTD,

150 Japan). Results were obtained in the CIE L*a*b* coordinates using the D65 standard 151 illuminant, and the $2^{\circ}$ standard observer (CIE 1931). The hue (h) and the chroma $\left(\mathrm{C}^{*}\right)$ 152 were calculated from them with the equations $h=\operatorname{atan}\left(b^{*} / a^{*}\right)$ and $C^{*}=\left(\left(a^{*}\right)^{2}+\left(b^{*}\right)^{2}\right)^{1 / 2}$ 153 (Ronda et al 2005). L* ranges from 0 (black) to 100 (white). The hue scale extends from $1540^{\circ}$ (red), $90^{\circ}$ (yellow), $180^{\circ}$ (green) to $270^{\circ}$ (blue). The chroma informs about the purity 155 of the colour: a near zero $\mathrm{C}^{*}$ value corresponds to a colour of low purity, near grey. On 156 the opposite high $C^{*}$ values mean colours of high purity near the pure spectral colours. 157 Colour determinations were made 5x5 times: bread crumb and crust colours were 158 checked at five different points on each bread and every point was measured five times.

1592.4 Sensory analysis

160 Sensory analysis was performed by a panel of ten semi-trained judges (four males and

six females aged 25-52), who scored the size and the uniformity of cells (crumb grain), the softness and chewiness of the crumb, the crumbliness of the crust, the taste and 
flavour (intensity and type), and the overall acceptability. Ratings were given to breads

164 wrapped in plastic bags and stored at room temperature for 24 hours. A semi-structured

165 scale from 1 (very little)-to 109 (very much)-was used. The attributes tested were: crumb grain ( $1=$ very large and inhomogeneous cells; $10=$ very small and homogeneous cells), crumb softness $(1=$ very hard; $10=$ very soft $)$, crumb chewiness $(1=$ very rubbery, requiring many bites to swallow; $10=$ very little rubbery), crust crumbliness $(1=$ very $\underline{\text { soft and annealed crust; } 10=\text { very crunchy crust) taste and flavour intensity (1: very little; }}$

10: very much). Additionally, Aan hedonic, - overall acceptability test was also included

in the sensory evaluation with an scale from 1 (dislike very much) to 10 (like very $\underline{\text { much). }}$

2.5. Statistical analyses

Multivariate statistical analysis of data (non-linear regression, stepwise regression analysis, and Pearson correlation analysis) was performed by using Statgraphics

177 Centurion v.6 program (Bitstream, Cambridge, MN, USA).

\section{Results and discussion}

Morpho-geometrical features, texture characteristics and sensory scores were measured to assess the quality and staling behaviour of fibre enriched GF breads made according to a Draper-Lin design. Retrieved instrumental physical parameters and sensory results were analysed for dependence on dough hydration and on viscous dietary fibres, and for correlations between dough and bread parameters (Table 5 and 6$)$.

\subsection{Effect on physical properties of fresh breads}

Analytical data on bread characteristics (Table 2)-were fitted to multiple regression equations using added principles (SFE, NE, BBG, WATER) as independent factors in order to estimate response surfaces of dependent analytical variables. Significant 
187 coefficients (95\% confidence interval) obtained from the stepwise regression fitting

188 model are included in Table 32 .

Specific volume and height/width rate

Loaf specific volume of the breads, that varied between 1.4 and $5.1 \mathrm{~mL} / \mathrm{g}$, and the loaf height/width ratio, that ranged $0.28-0.87$, exhibited similar trends, as could be expected in pan breads-where the width comes mainly determined by the mould size. The multiple regression equations obtained for height/width and specific volume explained the $99 \%$ and $91 \%$ of their variability, respectively (Table 42 ). Specific volume increased with WATER, SFE and BBG addition until a maximum-(Fig.1a and Fig.1b).. The positive coefficients of the linear terms and the negative coefficients of the quadratic ones of these three factors account for this evolution (Table 24). It should be noticed the non significant individual effect of NE $(p>0.05)$ on both the height and the specific volume of breads, were probably masked by the greater effect of the remaining design factors. The comparison by pairs of the experimental results obtained for each run (runs 1, 5, 10), allowed to confirm a significant increase $(p<0.05)$ in the specifie volume for increasing SFE amounts, provided remaining factors keep constant (data not shown). A significant positive interaction SFE*NE was observed, which means that $\mathrm{NE}$ enhanced the individual SFE effect on bread size. The effect of SFE on specific volume did not showed a significant dependence on dough water content, being $1.6 \%$ the dose that led to the maximum size of the bread-(Fig 1.a). From the multivariate regression equation the individual addition of $1.6 \%$ SFE to the dough with the minimum water content tested ( $70 \%$, equivalent to 0 level in the design) would nearly double the initial volume of the bread, passing from $1.4 \mathrm{~mL} / \mathrm{g}$ to $2.7 \mathrm{~mL} / \mathrm{g}$. For this $\mathrm{SFE}$ dose, a water increase from $70 \%$ to $90 \%$ would lead to an additional specific volume increase by 37\%. $90 \%$ of dough hydration-(equivalent to 20 level in the design)-led to the 
212 maximum bread specific volume, only dependent on BBG dose. The positive interaction 213 BBG*WATER (Table 42) indicates that water favors the effect of BBG on specific volume. However optimal water content of dough was not significantly dependent on SFE and NE.This fact establishes an important difference between the HPMC and BBG action on GF breads: BBG requires an important additional amount of water to show a beneficial effect on bread volume while HPMC acts even in adverse conditions of low 218 dough hydration showing an effect little dependent on dough water content. The effect are probably too consistent to get a certain development during proofing and subsequent baking (Ronda et al. 2013). BBG added to reduced-water doughs is unable of establishing cross-links or entanglements in the dough and, thus, dough structure cannot be developed. It was previously shown that BBG was unable to decrease the elastic compliance, $\mathrm{J}_{\mathrm{o}}$, at dough water content of $70 \%$ as HPMC did (Ronda et al 2013). But, in these conditions, it conferred the major effects on dough $G^{\prime}$ modulus. So that, BBG 233 addition to doughs only increased dough consistency, which made dough development 234 even more difficult, leading finally to a lower bread volume. Simultaneous addition of 235 water and BBG counteracted the single BBG effect through the significant positive 236 eoefficient of the interaction BBG*Water (Table 4), leading to an increase of $34 \%$ in 
237 bread volume compared to the maximum value obtained in BBG absence. In a previous 238 work (Ronda et al. 2013), a significant BBG*Water negative interaction on the elastic 239 modulus G' was found -for the same GF doughs, -allowing to relate the increase in bread volume with a decrease in dough consistency. The additional amount of water 241 required to get the maximum volume in bread increased at a rate of $5-6 \%$ per $1 \%$ 242 increase of BBG in dough formulation (flour basis in both cases): the dough hydration 243 needed for 2\% BBG would be $101 \%$, and for $3.5 \%$ BBG it would be $109 \%$. However, 244 the model predicts that without HPMC, even with the optimal dose of water, BBG 245 addition above $2.5 \%$ would start to decrease specific volume attaining a reduction of $246 \quad 24 \%$ at the maximum dose tested (3.9\%). Lazaridou et al. (2007) also observed a increase in bread volume by $1 \%-2 \% \beta$-glucan addition to dough. Hydrocolloids can improve dough development and gas retention by increasing dough viscosity (Houben, Hoechstoetter, and Becker 2012), but, there is an optimum value for the resistance to deformation. Too high values can cause a limited and slow expansion of the gas cells during proofing (Van Vliet et al 1992). From the negative sign of the quadratic coefficients of BBG and SFE, a dose leading to a maximum in bread height and specific volume may be retrieved. For HPMC, besides water retention properties associated to hydrophilic nature, hydrophobic groups induce additional properties including inereased interfacial activity within the dough system during proofing, and leading to gel network 
formation on heating over the breadmaking process. Such network structures serve to breads correspond to high doses of both SFE and water that gave poor quality breads with large gas cells (pictures not shown), in agreement with previous works (Haque and Morris 1994; McCarthy et al 2005; Nishita et al 1976) on high water GF breads.

Authors related pocket formation to a poor dough consistency due to an excess of water.

Up to a certain level, a soft consistency, as promoted by high water addition and limited amounts of hydrocolloids, seems to be advantageous, allowing a larger increase in volume. However, The excessive low consistency seems to cause the bubbles to become unstable, coalescing and resulting in large holes, after the crust has formed. In preliminary tests, with individual additions of either SFE or NE, it was found that the eccurrence of grain defect took place mainly for SFE breads and for water doses above $90 \%$, flour basis. Owing to the differences between the two types of HPMC used in the present work, defects could be related to the facility of crust forming that could be higher in the case of SFE (a semi-firm gel forming) than in NE (a weak gel forming) helping retain the large bubbles formed inside the crumb. With no exception, defects were only observed at water amounts above $90 \%$.

\section{Loss of weight}

The loss of weight during baking varied between $26 \mathrm{~g}$ and $41 \mathrm{~g}$, equivalent to $13 \%$ and $21 \%$ with respect to the initial amount of the baked dough-(Table 2). Table $4 \underline{2}$ shows that the loss of weight depended mainly on the water content of the dough. The 
quadratic coefficients) obtained for water-indicate that water loss increased up to a

maximum with increased dough water content (Table 42). When water content is low, increasing water results in an increase of the rate of water lost by evaporation. However, when the water content is high enough, above a critical amount an additional water increase did not lead to an additional increase in the loss of weight, since the amount of water lost during baking is determined by baking time that remained constant in this study. In absence of hydrocolloids the maximum loss of weight increase was $20 \%$ and took place at a dough hydration of $94 \%$. The marked effect of water content on loss of weight during baking probably masked the effect of the remaining design factors.

Although no significant coefficients were obtained for SFE in the multifactor regression, the comparison by pairs of the experimental results obtained for each run (see runs 3,7 and 9), allowed to confirm a significant increase $(p<0,05)$ in the loss of water during baking for increasing SFE amounts, provided remaining factors keep constant (data not shown). Similar results were previously obtained in hydrocolloidadded cake systems where HPMC was the only tested hydrocolloid that led to losses of weight in baking above the control cake (without hydrocolloid) (Gómez et al 2007). The individual addition of BBG to dough decreased the loss of weight during baking as indicated by the negative linear significant coefficient. At a flour hydration level of $70 \%$, the individual addition of $3.9 \% \mathrm{BBG}$ led to a $27 \%$ decrease in the loss of weight. This was probably due to the high water binding capacity of $\beta$-glucans in line with previous results (Brennan and Cleary 2007). However, the positive sign of the BBG*WATER interaction coefficient anticipates that an increase in dough water content decreased the individual ability of $\mathrm{BBG}$ to reduce the loss of weight during baking. Accordingly, the amount of water that led to the maximum loss of weight in BBG absence, $94 \%$, increased from $94 \%$ to $104 \%$ for $2 \%$ BBG and to $112 \%$ for $3.9 \%$ 
312 BBG. The mentioned dough hydration values that maximized the loss of weight during

313 baking also maximized the bread specific volume at each BBG addition.

\section{Texture of the bread}

315 Fresh bread crumb firmness varied from $1 \mathrm{~N}$ to $17 \mathrm{~N}$-(Table 2), which means a very

316 wide range of crumb hardness among tested breads. From the multiple regression study,

317 firmness was only significantly affected $(\mathrm{p}<0.05)$ by both water and BBG (Table

318 4Table 2). These only two factors could explain $91 \%$ of the variability of fresh bread

319 firmness, as indicates the $\mathrm{R}^{2}$ value included in Table 4Table 2 . The effect of cellulose

derivatives, which was detected by the analysis and comparison of individual experiences (Table 2), _was notably smaller than that associated to the other two ingredients. The individual addition of SFE led to a decrease in firmmess (runs 3, 7 and 9) that did not reach a significant level, while NE hardly showed any effect (runs 1, 5 and 10).-In absence of hydrocolloids, a significantly softer crumb was observed when the water content was increased from $70 \%$ to $90 \%$ _(Fig. 1c), _aas- it was observed reported earlier (McCarthy et al 2005). However, as can be predicted from the positive quadratic coefficient, additional amounts of water made crumb firmer again-. Firmness evolution could be explained in parallel to specific volume evolution. In fact, both properties showed a significant $(\mathrm{p}<0.001)$ negative correlation (see section 3.3 ). Water dosage that led to a minimum crumb firmness led to a concomitant maximum specific

volume. The same could be reported from the BBG effect. Additional amounts of water in absence of hydrocolloids, probably decreased dough consistency too much and hindered dough gas retention ability during proofing and baking. The fact would explain the decrease in bread volume and the consequent increase in bread firmness.

The individual effect of BBG can be concluded from the high positive coefficient of the

336 linear term of the regression equation (Table 4 Table 2). Accordingly, the individuat 

addition of BBG to the lowest hydration dough ( $70 \%$ flour basis, 0 level of water),

increased bread firmness more than seven times the initial firmness of bread (runs 2 and

8). Other authors observed that BBG increased firmness and decreased loaf volume of wheat breads (Brennan and Cleary 2007; Cavallero et al 2002). These effects were attributed to gluten dilution and to the BBG ability to binding appreciable amounts of water, making it less available for the development of gluten network. Similar results were obtained in GF bread related to the high water binding of BBG.However, the significant negative interaction between water and BBG explains that the simultaneous addition of BBG and water counteracted the individual effect of BBG. From the predictive model, addition of $4 \%$ BBG with water content above $95 \%$ would lead to crumbs with a similar firmness to those obtained with doses of cellulose derivative around $1.5 \%$. Fig. 1 shows the firmness of fresh breads and their evolution during nine days of storage. It allows the comparison of runs where only varies a factor while the rest are constant in the intermediate level of the range studied. The comparison of runs 7 and 11 shows the importance of dough hydration on BBG-enriched GF bread development (Table 2Fig. 1c).

Chewiness of breads ranged $0.3-5.8 \mathrm{~N}$ and varied in parallel as hardness did (Table 4Table 2). This could be expected as both textural properties are directly related. Cohesiveness (varied between 0.4 and 0.6 ) and springiness (ranged $0.6-0.9$ ), also involved in chewiness equation, showed minor effects. The resilience variation could not be correlated to the studied factors, probably due to the low values and the low variations observed between runs $(0.19-0.29)$. Springiness-or the after stress retarded recovery capacity, increased with single addition of BBG and/or water (see significant quadratic terms in Table 2):-_From the multivariate regression equation it could be predicted a $21 \%$ increase in crumb springiness obtained from low hydration dough 
362 (70\% water) with 3\% BBG and of 39\% for 3.9\% BBG. In absence of hydrocolloids the 363 increase of dough hydration from $80 \%$ to $110 \%$ led to springiness increases around a $36410 \%$ per each $10 \%$ increase in dough water content. The negative interaction 365 WATER*BBG (Table -4)-explains that the simultaneous increase of water and BBG in dough did not lead to the expected springiness as result of the sum of the individual

367 effect of Water and BBG.

368 Crumb and crust colour

369 Bread crust colour features showed bigger dependence on both hydrocolloid and water 370 contents, than bread crumb. Bread crusts showed lightness values that ranged 60-74\%.

371 The $a^{*}$ and $b^{*}$ coordinates varied between 2-13 and 19-30 respectively (see Fig 2)

372 leading to hue values that ranged of $64-84$ degrees, and chromas between-of $19-32$ 373 (Table 2 data not shown). Crust colours had higher $\mathrm{L}^{*}$ and $\mathrm{b}^{*}$ coordinates and lower $\mathrm{a}^{*}$ one than other authors found (Lazaridou et al, 2007) probably due to the use of corn as starch source in that work. This could be due to the presence of corn starch and multivariate regression equation (, from the positive quadratic term in Table $4 \underline{\text { Table } 2)}$ it would be predictable a $10 \%$ increase in bread crust Lightness for the maximum dough hydration of $110 \%$ (40 level) with respect to the lowest one $(70 \%)$. This was probably due to the reduction of Maillard reaction progress progress, main responsible for crust 
limited amount of water ( $70 \%$ water, flour basis) may affect the Maillard reaction. Aan

additional dose of SFE did not showed additional effect (Table 2), as predicts the

positive coefficient of the quadratic term shown in Table 4. Probably, above 2\%, SFE

hardly hydrated, and did not contribute to Maillard reaction notably. Single BBG

increased crust Lightness until $14 \%$ for the maximum dose tested, $3.9 \%$, in accordance

with the positive quadratic coefficient in the regression equation. The effect, also

observed in previous works (Lazaridou et al 2007), could be explained by the already

mentioned BBG water retention capacity during baking. that led to breads with

significantly lower weight loss. Simultaneous increase of BBG and Water led to darker

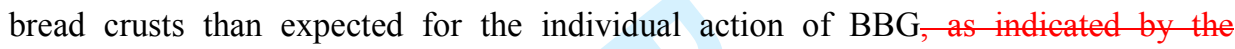
negative sign of the interaction (significant $B B G^{*}$ Water coefficient), concomitant with

the increase in baking water loss already mentioned in the previous section.

Bread crust hues, significantly -affected by all the factors studied (Table 2) corresponded to more yellowish $(\mathrm{h}=90)$ than reddish $(\mathrm{h}=0)$ colours. Bread crust hue was significantly affected by all the factors studied (Table 4).. It was observed that, in general, breads with lower crust hues (more reddish) had lower ltightness values. The This is probably due to a common origin of both crust colour attributes. The eireumstances that favoured Maillard reaction and decreased Lightness (SFE increase and the water/-BBG decrease) led to reddish and darker bread crust. Lazaridou et al (2007) also observed a tendency to yellowish in $1 \%$ oat $\beta$-glucan-added GF-bread crusts

407 (Lazaridou et al 2007). The crust hue was also significantly decreased by NE, although

408 in less extent than SFE, as it can be concluded from the lower linear and quadratic 409 regression coefficients (Table $4 \underline{\text { Table } 2}$ ).

410 Crumb colour, that mainly depends on the own ingredient colours, was not very affected 411 by the design factors due to the white-slight cream colour of added fibers.-_Only the 
412 chroma showed significant linear dependence on dough hydration and on viscous

413 dietary fibres with a $\mathrm{R}^{2}$ of $81.4 \%$. The $\mathrm{a}^{*}$ and $\mathrm{b} *$ coordinates of all bread crumbs ranged from -0.23 to +0.44 and from +7 to +12 respectively. These values corresponded to erumb hues (h) from 87 to 91 degrees, which correspond to yellow colours. The chroma $\left(C^{*}\right)$ values ranged 7 to 12 . Dough hydration had the highest effect on crumb chroma.

With no added hydrocolloids the maximum water content increase (40\%) led to 59\%

418 increase in the crumb cehroma (leading to the highest crumb-chroma values (more vivid colours)s). The presence of both types of HPMC counteracted the individual effect of water.

\section{Bread staling}

Bread staling was assessed by means of hardness evolution over 9 days (Figure 13 ). Experimental data were fitted to the Avrami equation (Armero and Collar 1998; Ronda and Roos 2011), and adjusted values were plotted leading to the continuous lines. Hardening during storage is expected as a result of moisture loss and starch retrogradation (Ronda et al 2011). Water was the factor with more prominent effect on

GF bread staling, as shown FiFig.ture 31c. This figure shows -shows that the bread

made with the lowest water content, $70 \%$ in flour basis reached, had after one storage day, a hardness ten times that of the bread with a $90 \%$ water content. After nine days of storage hardness was $80 \mathrm{~N}$, which represents an extremely high value. It should be noted that the curves in Figure 3 c correspond to constant concentrations of SFE, NE and BBG of $1.3 \%, 1.3 \%$ and $2 \%$ respectively. the bread made with a water content of $70 \%$ reached, after one storage day, a hardness ten times that of the bread with a $90 \%$ and the same hydrocolloid contents. After nine days of storage hardness was $84 \mathrm{~N}$, which represents an extremely high value. This demonstrates that hydrocolloids, with water restrictions, lead to unsuitable bread characteristics. The different effect of SFE and NE 

on bread hardening was also remarkable. Fig_tre- $13 \mathrm{~b}$ shows that SFE increase from $4380.1 \%$ to $2.5 \%$ led to significant depletion in bread hardness during storage, in spite of 439 1.3\% NE presence-in bread formulation. On the contrary, same changes in the 440 concentration of NE to a fixed concentration of $1.3 \%$ SFE hardly changed hardness

441 curves-(Figure 3b). SFE, therefore, showed a clearly better ability than NE to increase bread keepability. In previous studies it has been found that carboxymethyleellulose (CMC), added to GF doughs, controlled crumb hardening after three days storage (Lazaridou et al 2007).

As it can be seen in Fig. 1a, higher contents of BBG accelerated the aging of breads with $90 \%$ of water and $2.6 \%$ of HPMC $(1.3 \%$ SFE $+1.3 \%$ NE) As it can be seen in Figure 3a, higher contents of BBG-accelerated the aging of breads with $90 \%$ of water and $2.6 \%$ of HPMC $(1.3 \%$ SFE $+1.3 \% \mathrm{NE})$-_Other authors also observed this effect in breads added with $\beta$-glucan (Lazaridou et al 2007). Bread crumbs with 3.9\% of BBG were since the second day of storage significant firmer than those with lower BBG addition $(0.1 \%, 2 \%)$. This could be due to the This high dose of BBG would have required, attending its already commented-higher water necessities of this high $\mathrm{BBG}$ dose $(3.9 \%)$, minimize crumb firmness.. It is also noteworthy that the firming curves of breads with $2 \%$ and $3.9 \%$ of $\mathrm{BBG}$ did not reach a constant value in nine days, unlike the curve for breads with $0.1 \% \mathrm{BBG}$, which achieved in five days a stable value. $3.9 \% \mathrm{BBG}$ delayed the achievement of the maximum hardness during storage at $4^{\circ} \mathrm{C}$, generally attaint in $6-$ 8 days (Ronda and Roos 2011), probably due to the great water binding ability of BBG, controlling the loss of water during aging (Ronda et al 2011).

Fable 4 Table 2 shows the significant coefficients of the fitting model for the crumb hardening after one (short-term) and nine (long-term) days of storage at $4^{\circ} \mathrm{C}$. The 
462 regression study confirmed the individual effects of HPMC, BBG and Water on the

463

already mentioned crumb hardening. In short-term storage (one day), SFE effect on erumb hardness reduction was much more markedly than NE. Attending the regression equation a dose of $2 \%$ SFE would lead to a $40 \%$ reduction in the increment of firmness after one storage day. Individual effect of BBG was the opposite: Doses of 2\% and 3\% ted to an inerease in the hardness after one day of $17 \%$ and $38 \%$ respectively. In longterm-storage, water content interactions with hydrocolloids exerted the most marked effects on crumb hardness. BBG was responsible for a significant crumb hardening after nine days of storage, but the provision of additional water content to the formula reduced significantly the effect..

\subsection{Effect on bBread sensory quality}

Formatted: Font: Bold, Not Italic

The coefficients of the regression fitting models for the sensory attributes $\left(0.76 \leq \mathrm{R}^{2} \leq\right.$ 0.90) are compiled in Fable 4Table 2. Panelists scored crumb grain for both cell size and distribution, giving low ratings to big and heterogeneously distributed alveoli and high ratings to small and homogeneous gas cells (Table 3 ). SFE, BBG and wwater markedly affected crumb grain scores that varied between $2.4 \pm 0.5$-and $9.7 \pm 0.5$ in the scale 1-10. Negative coefficients of single BBG and/or Water (Fable 4 Table 2) suggest both factors increased alveoli size and crumb grain heterogeneity, although BBG quadratic coefficient supports that from $1.7 \%$ BBG addition crumb grain ratings became higher. SFE and Water showed a significant negative interaction on crumb grain scores, so that, high SFE dosages to softer doughs led to a prominent decrease in crumb grain ratings-(Table 3). Lower crumb grain scores were concomitant with the presence of big holes (pockets) in bread crumb, and unaccepted by panelists. Ratings for crumb softness and smoothness (Table 3)-were parallel and dependent on Water, BBG and SFE (Table 24). Increased water amounts up to $95-100 \%$ hydration in dough 
promoted significantly sensory ratings for crumb smoothnesssoftness. Higher hydration

levels did not provide any additional sensory smoothness-perception. BBG increasing single addition decreased smoothness_softness regardless dough water content. Simultaneous SFE presence helped BBG increase crumb smoothness-softness when added up to $2 \%$, flour basis. In fact, SFE counteracted BBG deleterious effect on crumb smoothness, so that SFE appears as a key ingredient in BBG-enriched GF-breads.

Overall acceptance of GF-breads, that varied between $2.2 \pm 0.4$, for Run 13 , and $7.6 \pm 0.4$, for Run 12, was only significantly affected $(\mathrm{p}<0.05)$ by water and BBG dosages. According to panelits' scores, overall acceptance was mainly/significantly dependent on crumb grain, and softness - and smoothness (Table 6Table 4). Breads with water formulation greater/equal than $90 \%$ (flour basis) deserved acceptance $(\geq 5 / 9)$, with the exception of Run 13, including $3.9 \%$ BBG. It should be noticed that the bread of Run 12 (3.34\% BBG, 0.45\% SFE, 2.15\% NE, 104\% WATER), was individually preferred (7.6 $\pm 0.4 / 9)$. The ratio $\mathrm{BBG} /$ Water of this formulation coincided with that predicted from the regression equations as a ratio capable of optimizing the bread quality leading to minimum bread firmness and maximum specific volume.

\subsection{Correlation between variables.}

Multivariate data handling of dough visco-elastic and bread quality parameters supplied useful information on the significantly correlated dough and bread characteristics. Fundamental and empirical dough rheological properties were reported in a previous work (Ronda et al 2013). Using Pearson correlation analysis, a range of correlation coefficients (r) (from 0.46 to 0.90 ) was obtained for the relationships between dough viscoelastic parameters and bread properties of fibre-supplemented rice-based matrices (Table 5Table 3). Table 6Table 4 reports Pearson correlation coefficients for the relationships between bread properties. Bread specific volume strongly correlated with 
512 dough viscosity at the steady state, $\eta_{0}$, obtained from creep tests $(\mathrm{p}<0.001, \mathrm{r}=-0.76)$.

513 Specific volume explicited a negative correlation with the elastic modulus at $1 \mathrm{~Hz}, \mathrm{G}_{1}$,,

$514(\mathrm{r}=-0.64)$ and positive with loss tangent, $\tan \delta$, and the exponents " $\mathrm{a}$ " and " $\mathrm{b}$ " $(\mathrm{r}=0.56$,

$515 \mathrm{r}=0.53, \mathrm{r}=0.56)$. The exponents, resulting from fitting power law to oscillatory

516 frequency sweep data, quantify the dependence of dough elastic and viscous moduli on

517 frequency. The higher the dependence of dynamic moduli on the frequency is, the

518 higher $\tan \delta$ and the greater the bread volume. This means that lower dough consistency

519 and less marked solid-like behaviour (within the range $0.19<\tan \delta<0.48$ ) favoured

520 dough development and bread volume. Baking weight loss was strongly correlated with

521 dough visco-elastic moduli, $\mathrm{G}_{1}{ }^{\prime}$ and $\mathrm{G}_{1}{ }^{\prime}{ }^{\prime},(\mathrm{p}<0.001 ; \mathrm{r}=-0.86 ; \mathrm{r}=-0.78)$, the viscosity at

522 the steady state, $\eta_{0},(\mathrm{p}<0.01 ; \mathrm{r}=-0.68)$ and the dough consistency or the force obtained

523 in a back extrusion test $(\mathrm{p}<0.001 ; \mathrm{r}=-0.82)$. The greater the dough consistency means

524 the higher the baking yields. This is probably due to the fact that fibres, substances

525 responsible for the dough consistency increase, were also good water-binding

526 macromolecules. Fresh bread crumb firmness and one- and nine-days stored bread

527 crumb hardening were strongly correlated to all visco-elastic dough properties. The

528 strongest positive correlation was obtained for fresh bread crumb firmness and the

529 elastic modulus, $\mathrm{G}_{1}{ }^{\prime}(\mathrm{p}<0.001 ; \mathrm{r}=0.79)$, and the viscosity at steady state, $\eta_{0},(\mathrm{p}<0.001 ; \mathrm{r}$

$530=0.88)$. The short- and long-term bread hardening exhibited similar relationships with

531 dough properties than initial firmness. Sensory scores related to crumb grain, crumb

532 softness and overall acceptance were strongly correlated $(p<0.001)$ with fundamental

533 and empirical dough rheological properties.

\section{4. Conclusions}


535 Draper-Lin small composite design for sampling allow to obtain useful information on 536 the significance of different hydrocolloid and water contents on breadmaking 537 performance and staling behaviour of GF rice-based complex/heterogeneous bread 538 matrices. Dough and bread characteristics strongly correlated supporting the key 539 influence of the restoration of dough visco-elasticity on the physico-chemical and 540 sensory achievement of GF final baked goods.

541 Single BBG by itself fails to mimic gluten visco-elasticity properly, but simultaneous 542 addition of either SFE or $\mathrm{NE}$, in doses around $1.6 \%$, contribute to bread improvement in terms of bigger volume and smoother crumb. The extent of methoxyl and hydroxypropyl substitution of HPMC markedly affects the gel strength. Weak gel forming NE led to harder and lower volume breads than semi-firm gel forming SFE did, probably ascribed to the ability of hydroxypropyl groups to form a stable solvate shell

547 in water restricting available water for starch to swell. It should be noticed that the 548 surplus of water needed to incorporate large amounts of BBG into bread favoured the formation of big holes (pockets) in bread crumb particularly when SFE is-was included into formulation. Visible holes could be probably related to the easier crust forming ability of SFE than NE helping retain the large bubbles formed inside the crumb. $\underline{A}$ dough hydration of $90 \%$ would be recommended to get the maximum volume of no BBG added breads. This optimal amount of water would increase at a rate of 5-6\% per $\underline{1 \%}$ increase in $\mathrm{BBG}$.

$3.3 \mathrm{~g}$ of $\underline{\mathrm{BBG} B B G}-(70 \%$ purity $)$ and $104 \mathrm{~mL}$ of water addition to $100 \mathrm{~g}$ rice flour provided sensorially accepted breads $(7.6 \pm 0.49)$ over 10$)$ with a theoretical 3 -glucan content of $1.24 \mathrm{~g} / 100 \mathrm{~g}$ GF bread. It would allow a daily intake of 3 ß-glucan providedwith a bread consumption of $240 \mathrm{~g} /$ day. A daily intake of $240 \mathrm{~g}$ of BBG enriched GF bread (four servings), in good accordance with the nutritional 
recommendation for bread consumption set between 200 and $300 \mathrm{~g}$ of bread per day

(Zessner et al 2011), is high enough to meet the requirements of the EFSA health claim

( $3 \mathrm{~g} /$ day), contributing a reduced blood cholesterol level.

An additional refined optimization of both water and quali and quantitative HPMC

addition for BBG concentrations from $3 \%$ to $4 \%$, is still recommendedComplementary

tests aimed at -establishing the real content and molecular weight of $-\not$-glucan in final

bread are still pending to assure the expected nutritional benefit of thisBBG

enrichement.to obtain healthy GF breads with improved organoleptic performance.

\section{Acknowledgement}

569 Authors gratefully acknowledge the financial support of the Spanish Institutions 570 Ministerio de Economía y Competitividad (Projects AGL2012-35088 and AGL2011571 22669) and Comunidad de Castilla y León (Project VA252A12-2), the laboratory of 572 Pharmacy (laboratory accredited according to ISO 17025) of the University of Basque 573 Country for the gluten analysis in barley $\beta$-Glucan extracts, and Cargill Company for

Anton, A. A. (2008). Improving the nutritional and textural properties of wheat flour tortillas. Cereal Research Communications, 36, 301-311.

578 Armero, E. \& Collar, C. (1998). Crumb firming kinetics of wheat breads with anti579 staling additives. Journal of Cereal Science, 28, 165-174.

580 enrichment of breads: A physicochemical and nutritional evaluation. Food Research

Formatted: Space Before: $12 \mathrm{pt}$, After: $0 \mathrm{pt}$ Formatted: Font: Bold 

glucan barley fractions in bread making and their effects on human glycemic response.

587 Journal of cereal science, 36, 59-66.

592 high fibre optimised formulations: Functional requirements. In: Intradfood Innovations 593 in Traditional Foods_(edited by P. Fito \& F. Toldrá). Pp. 879-882. London: Elsevier.

595 transglutaminase on the sensory, mechanical and crumb grain pattern of enzyme 596 supplemented fresh pan breads. Journal of Food Engineering, 70, 479-488.

Collar, C., Santos, E. \& Rosell, C. M. (2007). Assessment of the rheological profile of fibre-enriched bread doughs by response surface methodology. Journal of Food Engineering, 78, 820-826.

Draper, N. R. \& Lin, D. K. J. (1990). Small Responseresponse-SurfaceĐesignsdesigns. Technometrics, 32, 187-194.

EFSA. (2011). Scientific Opinion on the substantiation of health claims related to betaglucans from oats and barley and maintenance of normal blood LDL-cholesterol 608 Gill, S., Vasanthan, T., Ooraikul, B. \& Rossnagel, B. (2002). Wheat bread quality as 609 influenced by the substitution of waxy and regular barley flours in their native and 610 extruded forms. Journal of cereal science, 36, 219-237.

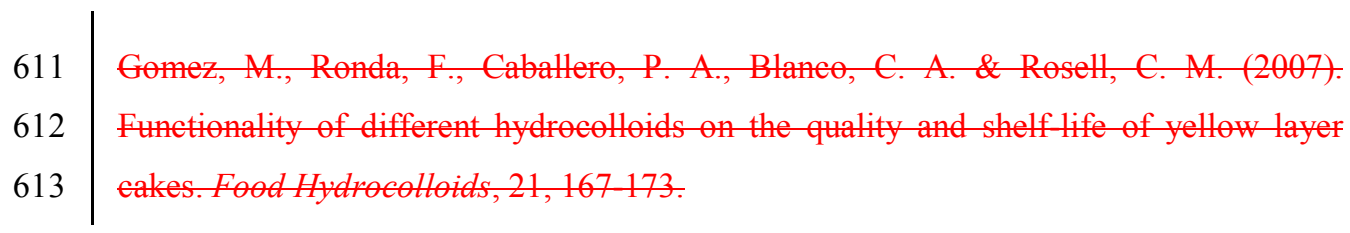


614

615 616 379-393. 638 fundamental and empirical rheological approach. Food Hydrocolloids, 32, 252-262.

639 Ronda, F. \& Roos, Y. H. (2011). Staling of fresh and frozen gluten-free bread. Journal 640 of cereal science, 53, 340-346. 

642 nondigestible oligosaccharides on the quality of sugar-free sponge cakes. Food 643 Chemistry, 90, 549-555.

644 Ronda, F., Caballero, P. A., Quilez, J. \& Roos, Y. H. (2011). Staling of frozen partly 645 and fully baked breads. Study of the combined effect of amylopectin recrystallization 646 and water content on bread firmness. Journal of cereal science, 53, 97-103.

647 Sabanis, D., Lebesi, D., Tzia, C. (2009). Effect of dietary fibre enrichement on selected 648 properties of gluten-free bread. Food Science and Technology, 42, 1380-1389.

649

650 651 Blackwell.

652

653

654

655 656

Thomson, T. (2009). The nutritional quality of gluten-free foods. In: Gluten-Free Food Science and Technology_(edited by E. Gallagher). Pp. 45-51. New Delhi: Wilet-

Van Vliet, T., Janssen, A. M., Bloksma, A. H. \& Walstra, P. (1992). Strain-Hardening hardening of Dough $\underline{\text { dough as a Requirement requirement for Gas gas }}$ Retentionretention. Journal of Texture Studies, 23, 439-460.

Wood, P. J. (2010). Oat and Rye beta-Glueanglucan: Properties and Funetionfunction. Cereal Chemistry, 87, 315-330.

Zessner, M., Helmich, K., Thaler, S., Weigl, M., Wagner, K. H., Haider, T., Mayer, M. M. \& Heigl, S. (2011). Ernährung und Flächennutzung flächennutzung in Österreich. Öwaw, 5/6, 95-104. 

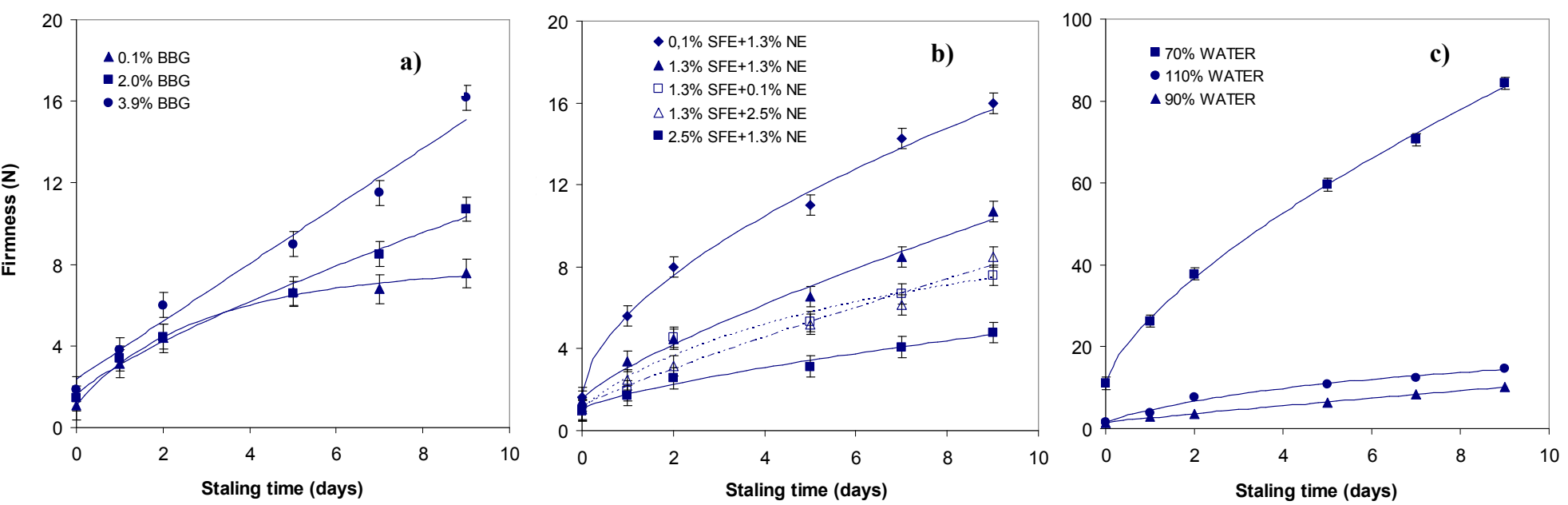

Figure 13: Crumb Ffirmness evolution during 9 days of storage time ofat $4^{\circ} \mathrm{C}$ of breads with different amounts of BBG (runs 5,7 and 13) (a) HPMC (runs $1,3,7,9$ and $10-(b)$ and Water (runs 7,11 and 14$)$ (c). Intermediate doses of dietary fibres $(1.3 \% \mathrm{SFE} ; 1.3 \% \mathrm{NE} ; 2 \%$ BBG) and water (90\% WATER) were used as constant factors. Values of firmness are the average of four replicates and error bars represent standard deviation. 
Table 1: Draper-Lin small composite design for sampling

\begin{tabular}{|c|c|c|c|c|}
\hline Run & SFE & NE & BBG & WATER \\
\hline 1 & 0 & -1.4142 & 0 & 0 \\
\hline 2 & -1 & -1 & -1 & -1 \\
\hline 3 & -1.4142 & 0 & 0 & 0 \\
\hline 4 & 1 & -1 & 1 & 1 \\
\hline 5 & 0 & 0 & -1.4142 & 0 \\
\hline 6 & 1 & 1 & 1 & -1 \\
\hline 7 & 0 & 0 & 0 & 0 \\
\hline 8 & -1 & -1 & 1 & -1 \\
\hline 9 & 1.4142 & 0 & 0 & 0 \\
\hline 10 & 0 & 1.4142 & 0 & 0 \\
\hline 11 & 0 & 0 & 0 & -1.4142 \\
\hline 12 & -1 & 1 & 1 & 1 \\
\hline 13 & 0 & 0 & 1.4142 & 0 \\
\hline 14 & 0 & 0 & 0 & 1.4142 \\
\hline 15 & 0 & 0 & 0 & 0 \\
\hline 16 & 1 & -1 & -1 & 1 \\
\hline 17 & -1 & 1 & -1 & 1 \\
\hline 18 & 1 & 1 & -1 & -1 \\
\hline 19 & 0 & 0 & 0 & 0 \\
\hline
\end{tabular}

Design factors are: hydroxypropylmethylcellulose semi-firm gel forming HPMC SFE 4000 (SFE). hydroxypropylmethylcellulose weak gel forming HPMC NE 4000 (NE). Barley Beta-Glucan (BBG). and water addition (WATER). -1.4142. -1. 0. 1. and 1.4142 indicate coded levels of design factors; axial distance from the center points: 1.1 .4142 . 


\section{Page 31 of 33}

International Journal of Food Science \& Technology

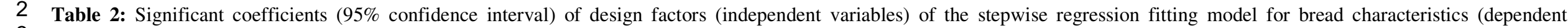
3 analytical variables).

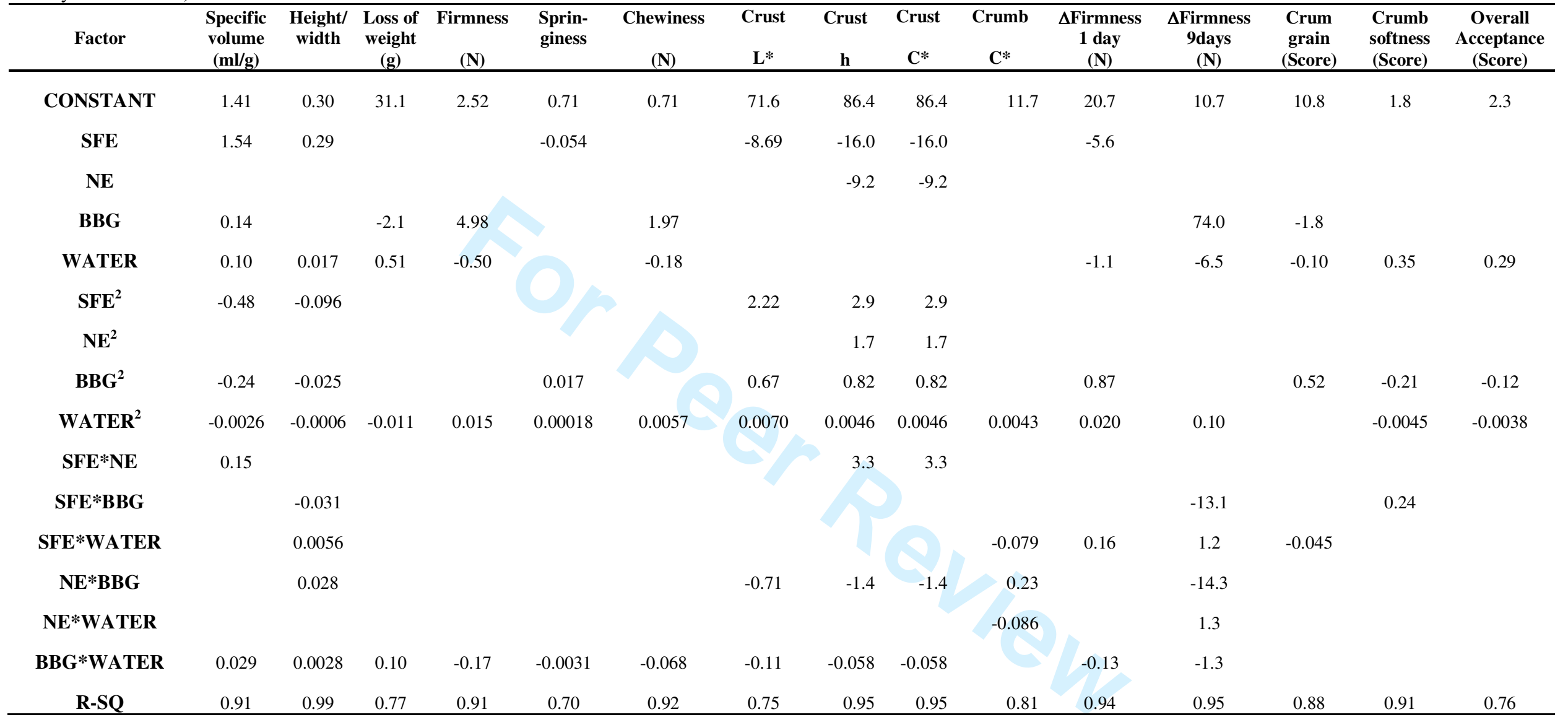

Independent variables: SFE= HPMC SFE 4000; NE= HPMC NE 4000; BBG: Barley Beta-Glucan; WATER= water content above 70\%. Blanks correspond to no significant effects at level of 5\%; R-SQ adjusted square coefficient of the fitting model 
Table 3: Pearson product moment correlations between pair of variables measured in dough and bread. These correlation coefficients range between -1 and +1 and measure the strength of the linear relationship between the variables. The rheological properties correspond to the fitting of experimental oscillatory measurements to power law model $\left(G^{\prime}=G^{\prime}{ }_{1} \cdot \omega^{a} ; G^{\prime}{ }^{\prime}=G^{\prime}{ }_{1} \cdot \omega^{b} ; \tan \delta=(\tan \delta)_{1} \cdot \omega^{c}\right)$. Creep test results to the 6-parameter Burgers model (where $\mathrm{J}_{\mathrm{o}}$ and $\mu_{\mathrm{o}}$ are the instantaneous compliance and the steady state viscosity respectively); Consistency is the firmness measured in an extrusion empirical test (Ronda et al. 2013).

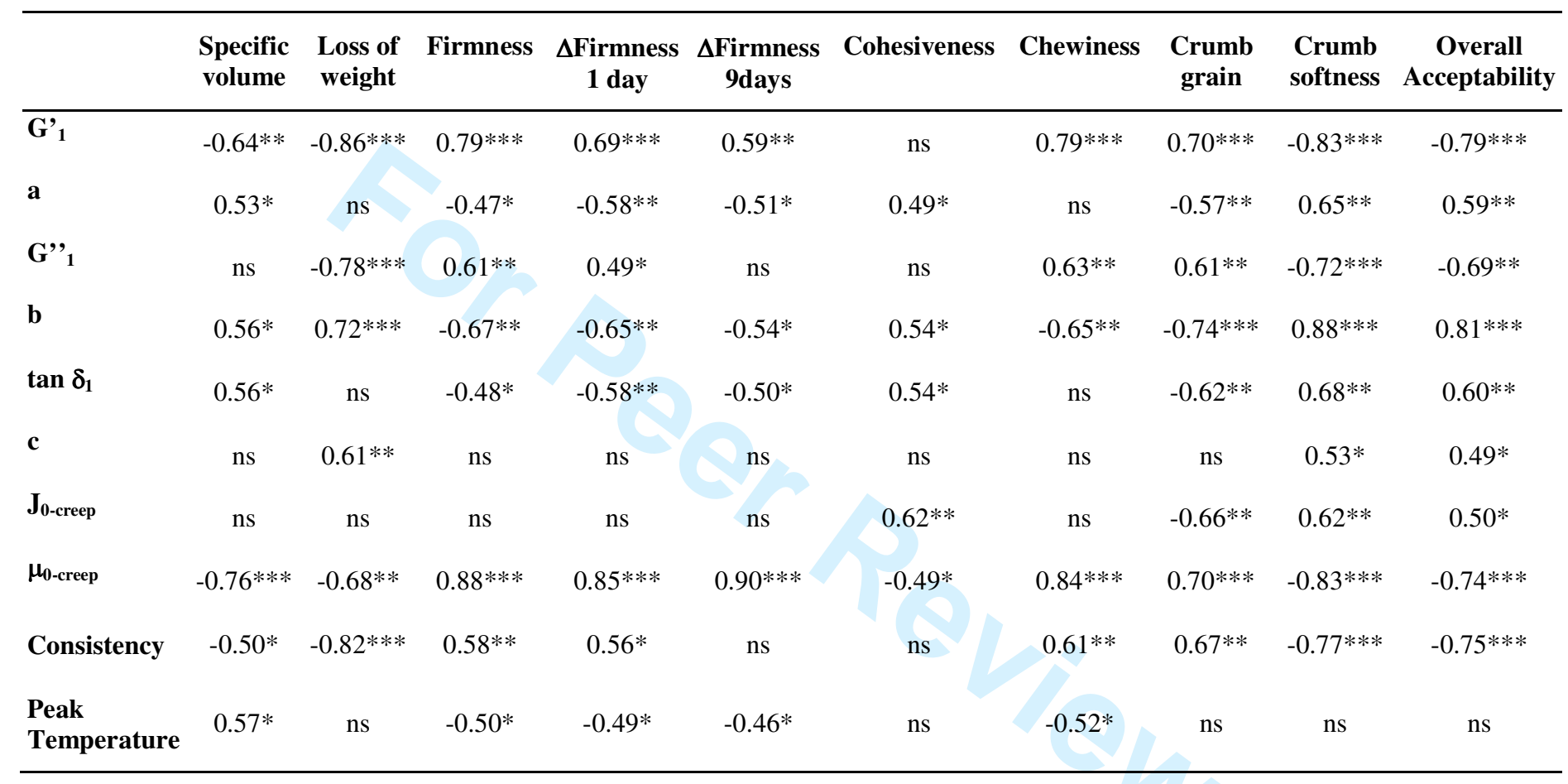

Asterisks indicate the P-value which tests the statistical significance of the estimated correlations. ${ }^{*} \mathrm{p}<0.05$ (statistically significant non-zero correlations at the $95.0 \%$ confidence level); ** $\mathrm{p}<0.01$ (at the $99.0 \%$ confidence level); *** $\mathrm{p}<0.001$ (at the $99.9 \%$ confidence level). ns: Not significant $(\mathrm{p}>0.05$ ) 
Table 4: Pearson product moment correlations between pair of variables measured in bread. These correlation coefficients range between -1 and +1 and measure the strength of the linear relationship between the variables.

\begin{tabular}{|c|c|c|c|c|c|c|c|c|c|c|c|}
\hline & $\begin{array}{l}\text { Loss of } \\
\text { weight }\end{array}$ & Firmness & $\begin{array}{c}\Delta \text { Firmness } \\
1 \text { day }\end{array}$ & $\begin{array}{c}\Delta \text { Firmness } \\
\text { 9days }\end{array}$ & Chewiness & $\begin{array}{c}\text { Crust } \\
\text { Lightness }\end{array}$ & $\begin{array}{l}\text { Crust } \\
\text { hue }\end{array}$ & $\begin{array}{c}\text { Crust } \\
\text { Chroma }\end{array}$ & $\begin{array}{c}\text { Crumb } \\
\text { grain }\end{array}$ & $\begin{array}{c}\text { Crumb } \\
\text { softness }\end{array}$ & $\begin{array}{c}\text { Overall } \\
\text { Acceptance }\end{array}$ \\
\hline $\begin{array}{l}\text { Specific } \\
\text { volume }\end{array}$ & $0.83 * * *$ & $-0.80 * * *$ & $-0.83 * * *$ & $-0.72 * * *$ & $-0.80 * * *$ & $-0.49 *$ & $-0.56^{*}$ & $0.62 * *$ & $-0.72 * * *$ & $0.73 * * *$ & $0.73 * * *$ \\
\hline $\begin{array}{l}\text { Loss of } \\
\text { weight }\end{array}$ & & $-0.75 * * *$ & $-0.74 * * *$ & $-0.55^{*}$ & $-0.77 * * *$ & ns & $\mathrm{ns}$ & ns & $-0.75 * * *$ & $0.76^{* * *}$ & $0.83 * * *$ \\
\hline Firmness & & & $0.92 * * *$ & $0.91 * * *$ & $0.99 * * *$ & ns & $\mathrm{ns}$ & $-0.61 * *$ & $0.55^{*}$ & $-0.73 * * *$ & $-0.62 * *$ \\
\hline $\begin{array}{l}\Delta \text { Firmness } \\
1 \text { day }\end{array}$ & & & & $0.87 * * *$ & $0.90 * * *$ & ns & $0.53^{*}$ & $-0.65^{* *}$ & $0.64 * *$ & $-0.81 * * *$ & $-0.71 * * *$ \\
\hline $\begin{array}{l}\Delta \text { Firmness } \\
9 \text { days }\end{array}$ & & & & & $0.85^{* * *}$ & $\mathrm{~ns}$ & $0.65^{* *}$ & $-0.61 * * *$ & $0.46^{*}$ & $-0.64 * *$ & $-0.51 *$ \\
\hline Chewiness & & & & & & ns & ns & $-0.57 *$ & $0.55^{*}$ & $-0.71 * * *$ & $-0.62 * *$ \\
\hline $\begin{array}{l}\text { Crust } \\
\text { Lightness }\end{array}$ & & & & & & & $0.72 * * *$ & $-0.56^{*}$ & $\mathrm{~ns}$ & $\mathrm{~ns}$ & ns \\
\hline $\begin{array}{l}\text { Crust } \\
\text { hue }\end{array}$ & & & & & & & & $-0.78 * * *$ & $\mathrm{~ns}$ & ns & ns \\
\hline $\begin{array}{l}\text { Crust } \\
\text { Chroma }\end{array}$ & & & & & & & & & ns & $\mathrm{ns}$ & ns \\
\hline $\begin{array}{l}\text { Crumb } \\
\text { grain }\end{array}$ & & & & & & & & & & $-0.83 * * *$ & $-0.83 * * *$ \\
\hline $\begin{array}{l}\text { Crumb } \\
\text { softness }\end{array}$ & & & & & & & & & & & $0.91 * * *$ \\
\hline
\end{tabular}

Asterisks indicate the P-value which tests the statistical significance of the estimated correlations. *p $<0.05$ (statistically significant non-zero correlations at the $95.0 \%$ confidence level); $* * \mathrm{p}<0.01$ (at the $99.0 \%$ confidence level); *** $\mathrm{p}<0.001$ (at the $99.9 \%$ confidence level). ns: Not significant $(\mathrm{p}>0.05)$ 